\title{
Diagnostic values of serum cathepsin B and $D$ in patients with nasopharyngeal carcinoma
}

\author{
Gongjun Tan ${ }^{1,2}$, Qianxu Liư², Xiaowei Tang ${ }^{3}$, Ting Kang ${ }^{4}$, Yuejin Li ${ }^{2}$, Jinping Lu², Xiaoming Zhao ${ }^{2}$ \\ and Faqing Tang ${ }^{1,2^{*}}$
}

\begin{abstract}
Background: The diagnostic and prognostic significance of increased cathepsin B (CTSB) and cathepsin D (CTSD) concentration in the serum of cancer patients were evaluated for some tumor types. High expression of CTSD and CTSB was detected in biopsy tissues from nasopharyngeal carcinoma (NPC). However, whether CTSD and CTSB serve as diagnostic and prognostic markers of NPC remains unclear.

Methods: Serum samples were collected from 40 healthy volunteers and 80 NPC patients enrolled in the study. CTSB and CTSD in the serum samples were detected using enzyme-linked immunosorbent assay (ELISA). Concomitantly, the relationship between CTSB and CTSD concentrations and clinicopathological prognosis was assessed. The sensitivity and specificity of the two components in the diagnosis of NPC were evaluated in 80 NPC patients.

Results: ELISA analysis showed that in the sera obtained from NPC patients, the CTSB concentration was $12.5 \pm$ $3.5 \mathrm{mg} / \mathrm{L}$ (median, $12.4 \mathrm{mg} / \mathrm{L}$ ), and the CTSD concentration was $15.7 \pm 8.7 \mathrm{mg} / \mathrm{L}$ (median, $14.7 \mathrm{mg} / \mathrm{L}$ ). CTSB and CTSD levels were significantly higher in the NPC patient population compared to the healthy control population ( $p=0.001 ; p=0.001$, respectively). The presence of CTSB and CTSD in the serum of the patients with NPC correlated with the tumor node metastasis (TNM) scores $(p=0.001)$. Other parameters were not identified to be of significance. Receiver operating characteristic (ROC) analysis showed that a cut off CTSB concentration of $12.4 \mathrm{mg} / \mathrm{L}$ had $61.9 \%$ sensitivity and $63.2 \%$ specificity in the prediction of progression-free survival (Area under the curve $(A \cup C)=0.525 ; 95 \% \mathrm{Cl}, 39.7-65.2 ; p=0.704)$; whereas a cut off CTSD concentration of $14.7 \mathrm{mg} / \mathrm{L}$ had $66.7 \%$ sensitivity, and $58.5 \%$ specificity (AUC $=0.552 ; 95 \% \mathrm{Cl}, 42.3-68.1 ; p=0.42$ ).
\end{abstract}

Conclusions: Serum CTSB and CTSD concentrations were found to have a diagnostic value in NPC. However, the CTSB and CTSD serum levels had no prognostic role for the outcome in NPC patients.

Keywords: Biomarker, Diagnostic value, Nasopharyngeal carcinoma, Cathepsin B, Cathepsin D

\section{Background}

Nasopharyngeal carcinoma (NPC) is one of the most common malignancies in Southern China, Southeastern Asia, and Northern Africa. The lowest prevalence of NPC is observed in white populations from Europe and the United States [1]. Most patients are initially diagnosed at a

\footnotetext{
* Correspondence: tangfaqing33@hotmail.com

'Department of Clinical Laboratory and Medical Research Center, Xiangya Hospital, Central South University, 87 Xiangya Road, Changsha 410008Hunan, China

${ }^{2}$ Department of Clinical Laboratory and Medical Research Center, Zhuhai People's Hospital, Zhuhai Hospital of Jinan University, 79 Kangning Road, Zhuhai 519000Guangdong, China

Full list of author information is available at the end of the article
}

late stage, resulting in high mortality rates. Radiotherapy is currently the primary treatment modality for patients with NPC. Although the tumor node metastasis (TNM) staging system is currently the most powerful prognostic factor of NPC, patients with the same TNM stage undergoing similar treatment regimens show variable clinical outcomes [2]. However, locoregional recurrence and distant metastasis following radiotherapy still have deleterious effects on the survival rates of patients with NPC [3]. Individualized therapy is needed to improve long-term survival and quality of life [2]. Determining clinical and biological characteristics of NPC, and identifying markers 
that can predict prognosis might make individualization of treatment possible [4].

Cathepsins are a class of globular proteases that were initially described as intracellular peptide hydrolases, although several cathepsins also have extracellular functions [5]. Cathepsins become proteolytically activated when attached to other cell surface proteins [6]. This extracellular activity enables cancer cell invasion of surrounding tissues, blood, and lymph vessels, and metastasis to distant sites. Numerous studies have shown a correlation between cathepsin proteolytic activity and neoplastic transformation, tumor invasion, and metastasis through the destruction of extracellular matrix components and basement membranes [7]. Elevated cathepsin B (CTSB) and cathepsin D (CTSD) levels were detected in primary and metastatic tumor tissues of various cancer types [8]. Elevation of CTSB and CTSD levels in biological fluid has been observed in patients with inflammatory diseases and many cancers. CTSB and CTSD are members of the cathepsin family. CTSB is a lysosomal cysteine protease of the papain family of enzymes that function as endopeptidases and exopeptidases [9]. CTSD is from the family of aspartic proteases that function in intracellular catabolism at lysosomal compartments; other physiological effects include hormone and antigen processing $[10,11]$. While they can both degrade laminin, fibronectin, collagen, and other extracellular matrix components, and promote the formation of tumor blood vessels [12], there is no similarity in their amino acid sequences. Our previous studies showed that CTSB and CTSD are highly expressed in NPC metastatic tissues, and they increased cell motility and invasion, and promoted NPC tumor metastasis [13]. Therefore, we speculate that CTSB and CTSD may be biomarkers of NPC metastasis, and could be of prognostic significance for NPC metastasis. In this study, we detected the concentrations of CTSB and CTSD in the sera of NPC patients and healthy controls using ELISA, analyzed the correlation of CTSB and CTSD concentrations with NPC progression, and evaluated the diagnostic significance of serum CTSB and CTSD concentrations for NPC patients. Our results showed that serum CTSB and CTSD concentrations are of diagnostic significance for NPC patients; however, they have no prognostic value in NPC patient outcomes.

\section{Methods}

The study protocol was approved by Ethical Committee at Zhuhai Hospital of Jinan University, and the written informed consent was obtained from each patients and control subjects. The participants declared that their sample and data are only used for this research in the signed informed consent, and the data could not be used in other research. The procedures of the study followed were in accordance with the ethical standards of the committee on human experimentation of the institution.

\section{Patient samples}

Eighty consecutive NPC patients (52 men and 28 women) with a median age of 45 years (range, 16-77), admitted to Sun Yat-sen University Cancer Center between February 2011 and December 2012, were enrolled in this study. The patients' characteristics with respect to age, sex, and ethnic origin were recorded. All of the enrolled patients were uniformly given a routine diagnostic work up constituted of a detailed clinic head and neck examination, nasopharyngoscopy, and the histological and cytological examination of tumor tissue. Tumor histology and stages were classified according to the World Health Organization (WHO) classification and the TNM staging system of Union for International Cancer Control (UICC), respectively. The concentrations of bilirubin, hepatic enzymes, and markers of renal function were also analyzed. The control group consisted of 40 healthy relatives of the NPC patients (20 men and 20 women), with a median age of 45 years (range, 20-78 years), and no known diseases.

\section{Sample collection}

Blood samples were obtained from fasting subjects under standard conditions. During the study period, blood samples were collected by venipuncture from the healthy control and patient population everyday between 9: 00 AM and 10: 00 AM. Samples were clotted at $4-8{ }^{\circ} \mathrm{C}$, and then centrifuged at $3000 \mathrm{rpm}$ for $10 \mathrm{~min}$. The collected serum was distributed in aliquots of $500 \mu \mathrm{L}$ each, and stored at $-80^{\circ} \mathrm{C}$ until required.

\section{Reagents and kits}

Chemical reagents used in the preparation of phosphatebuffered saline (PBS), including $\mathrm{NaCl}, \mathrm{KCl}, \mathrm{Na}_{2} \mathrm{HPO}_{4}$, and $\mathrm{KH}_{2} \mathrm{PO}_{4}$, were purchased from Sigma-Aldrich (St.Louis, MO). These salts were dissolved in $800 \mathrm{~mL}$ of distilled water, and the $\mathrm{pH}$ adjusted to 7.4 with $\mathrm{HCl}$. Distilled water was added up to a final volume of $1 \mathrm{~L}$. The resultant $1 \times$ PBS should have a final concentration of $10 \mathrm{mM} \mathrm{PO}_{4}^{3-}, 137 \mathrm{mM} \mathrm{NaCl}$, and $2.7 \mathrm{mM} \mathrm{KCl}$. CTSB and CTSD enzyme-linked immunosorbent assay (ELISA) kits were purchased from Wuhan Eiaab Science Co. Ltd (Hubei, China).

\section{Measurement of CTSB and CTSD}

Using commercial CTSB and CTSD ELISA kits, CTSB and CTSD detection was performed according to the manufacturer's instructions. All samples were detected simultaneously. Serum concentrations of CTSB and 
CTSD were determined using standard curves and expressed as units per liter $(\mathrm{mg} / \mathrm{L})$. The linear ranges for CTSB and CTSD were $0-50 \mathrm{mg} / \mathrm{L}$ and $0-100 \mathrm{mg} / \mathrm{L}$, respectively.

\section{Statistical analysis}

The group differences were assessed using nonparametric tests. Median concentrations of CTSB and CTSD in the patient and healthy control groups were compared using Mann-Whitney $U$ test. Using the median values as cut off values for both markers, the receiver operating characteristic (ROC) analysis was performed. The significance of serum CTSB and CTSD concentrations in the prediction of progression-free survival (PFS) was evaluated by univariate analysis. Survival curves were constructed using the Kaplan-Meier method, and compared with log-rank tests. Factors predictive of relapse were analyzed by both univariate and multivariate analyses using a Cox proportional hazards model. Multivariate $p$ values were used to characterize the independence of these factors. The relationship between survival time and each independent factor was quantified by calculating the $95 \%$ confidence interval (CI). All $p$ values were two-sided, and $p$ values $<0.05$ were considered statistically significant. All statistical analyses were performed using SPSS 17.0.

\section{Results}

The median ages for the control and patient groups were 40 years (range, 23-71) and 52.5 years (range, 18-86), respectively. Among 80 patients, 22 (27.5\%) had stage II, 25 (31.25 \%) had stage III, and 33 (41.25\%) had stage IV diseases. Seventy patients $(87.5 \%)$ were diagnosed with undifferentiated carcinoma. Sixty-eight patients (85.0 \%) completed radical radiotherapy. Demographic, disease, treatment, and relapse characteristics of the patients are summarized in Table 1.

CTSB and CTSD were identified to be associated with various cancer types [8], and we previously showed that CTSB and CTSD play important roles in NPC development and metastasis [13]. To evaluate the value of CTSB and CTSD in the clinical diagnosis of NPC, at first, CTSB and CTSD were detected in the sera of NPC patients and the healthy population. The results showed that median serum CTSB was $12.5 \pm 3.5 \mathrm{mg} / \mathrm{L}$ in the NPC group and $2.5 \pm 1.4 \mathrm{mg} / \mathrm{L}$ in the healthy control; while CTSD concentration was $15.7 \pm 8.7 \mathrm{mg} / \mathrm{L}$ in the NPC group and $2.7 \pm 0.8 \mathrm{mg} / \mathrm{L}$ in the healthy control population. CTSB and CTSD were significantly higher in the NPC group than in the healthy control group $(p<0.001)$ (Table 2$)$.

The associations between CTSB and CTSD concentrations and patients' clinical characteristics were further analyzed. The data showed a significant relationship
Table 1 Demographic, disease, treatment and relapse characteristics of the patients with nasopharyngeal carcinoma

\begin{tabular}{ll}
\hline Variables & Number (\%) \\
\hline Median Age (min-max) & $45(16-77)$ \\
Men & $52(65)$ \\
Women & $28(35)$ \\
Stage & \\
II & $22(27.5)$ \\
III & $25(31.25)$ \\
IV & $33(41.25)$ \\
Pathology & \\
Differentiated & $10(12.5)$ \\
Undifferentiated & $70(87.5)$ \\
Treatment & \\
Yes & $68(85)$ \\
No & $12(15)$ \\
Relapse & \\
None & $56(70)$ \\
Distant & $12(15)$ \\
Locoregional & $6(7.5)$ \\
Distant and Locoregional & $6(7.5)$ \\
\hline
\end{tabular}

between CTSB and CTSD concentrations and TNM grade ( $p=0.0001$ and $p=0.002$, respectively). We did not observe any significant relationships between CTSB and CTSD concentrations, and patient age, sex, viral capsid antigen (VCA)-IgA, alcohol intake, and smoking status (Table 3).

The significance of CTSB and CTSD concentrations in the prediction of NPC progression-free survival (PFS) was assessed. The median follow-up period for NPC patients was 24 months. During follow-up, 24 patients developed disease recurrence, including 12 with distant metastasis, 6 with local regional relapse, and 6 with both (Table 1). The 1 -year PFS rate was $78.5 \%$, whereas the median PFS was 25.6 months (min-max: 1.8-46.5). For each of the two parameters, overall survival was compared in patients with levels below, and equal to or above the median. We used ROC analysis to evaluate the prognostic significance of CTSB and CTSD concentrations for PFS, and identified that a CTSB cutoff value of $12.4 \mathrm{mg} / \mathrm{L}$ had a sensitivity of $61.9 \%$ and a specificity of $63.2 \%$ (AUC $=0.525 ; 95 \%$ CI, 39.7-65.2; $p=0.704$ ) (Fig.1), whereas a CTSD cutoff value of $14.7 \mathrm{mg} / \mathrm{L}$ had a sensitivity of $66.7 \%$ and a specificity of $58.5 \%(\mathrm{AUC}=0.552 ; 95 \% \mathrm{CI}, 42.3-68.1 ; p=0.42)$ (Fig.2). However, they were not significant prognostic factors for PFS. Univariate analysis also showed that clinical stage $(p=0.5)$, patient age $(p=0.7)$, sex $(p=$ 
Table 2 Serum CTSB and CTSD concentrations of the patients with nasopharyngeal carcinoma and the healthy population

\begin{tabular}{|c|c|c|c|c|c|}
\hline & \multicolumn{2}{|c|}{ Patients $(n=80)$} & \multicolumn{2}{|c|}{ Controls $(n=40)$} & \multirow[t]{2}{*}{$p$ value } \\
\hline & Mean $( \pm S D)$ & Median (min-max) & Mean $( \pm S D)$ & Median (min-max) & \\
\hline CTSB (mg/L) & $12.5 \pm 3.5$ & $12.4(4.4-25.6)$ & $2.5 \pm 1.4$ & $2.1(0.6-5.0)$ & 0.001 \\
\hline CTSD (mg/L) & $15.7 \pm 8.7$ & $14.7(2-42.3)$ & $2.7 \pm 0.8$ & $2.8(1.3-3.7)$ & 0.001 \\
\hline
\end{tabular}

Notes: CTSB cathepsin, B CTSD cathepsin D, SD standard deviation

$0.9)$, VCA-IgA $(p=0.7)$, and smoking status $(p=0.9)$ were not associated with PFS. Similarly, multivariate analysis showed that serum CTSB or CTSD concentrations were not of prognostic significance for PFS (Table 4).

\section{Discussion}

A number of clinical studies have been performed to evaluate the diagnostic and prognostic significance of elevated CTSB and CTSD concentrations in tumor cytosols [14]. It was shown that patients with higher concentration or increased proteolytic activity of CTSB in primary lung tumors exhibited significantly higher risk of recurrence or death compared to patients with a low concentration of the enzyme [15]. CTSD and CTSB activities in the sera of patients with urothelial bladder cancer were identified to be directly proportional to disease severity, and were significantly higher compared to those of the control group [16]. The elevation of CTSB and CTSD concentrations was shown in the sera of patients with some tumor types, including colorectal cancer, and bladder cancer [16, 17]. We previously showed that CTSD and CTSB are highly expressed in NPC tissue biopsies [13]. However, whether CTSD and CTSB serve as diagnostic markers of NPC has not been investigated. In this study, we detected the CTSD and CTSB concentrations in the sera of NPC patients and healthy controls, and analyzed the relationship between CTSB and CTSD concentrations and the occurrence of NPC. Our data showed that the CTSB and CTSD concentrations were higher in the sera of the NPC patients than in those of the healthy controls. Based on these results, we speculated that CTSB and CTSD concentrations might increase with the progression of NPC. In order to validate, we comparatively assessed the relationship of CTSB and CTSD with the NPC TNM grade for significance. The results showed that the concentrations of CTSB and CTSD increase with increasing TNM grade.

Table 3 Relationships between serum CTSB and CTSD concentrations and clinicopathological characteristics of patients with nasopharyngeal carcinoma

\begin{tabular}{|c|c|c|c|c|c|}
\hline \multirow[t]{2}{*}{ Variables } & \multirow[t]{2}{*}{ Number } & CTSB (mg/L) & \multirow[t]{2}{*}{$P$ value } & CTSD (mg/L) & \multirow[t]{2}{*}{$p$ value } \\
\hline & & Median (min-max) & & Median (min-max) & \\
\hline \multicolumn{6}{|l|}{ Sex } \\
\hline Men & 52 & $12.4(4.4-25.6)$ & \multirow[t]{2}{*}{0.823} & $16.6(2-42.3)$ & \multirow[t]{2}{*}{0.153} \\
\hline Women & 28 & $12.0(8.0-19.0)$ & & $2.7(4.6-29.1)$ & \\
\hline \multicolumn{6}{|l|}{ Age, years } \\
\hline$\leq 45$ & 42 & $11.9(4.4-25.6)$ & \multirow[t]{2}{*}{0.855} & $15.3(3.8-42.3)$ & \multirow[t]{2}{*}{0.086} \\
\hline$>45$ & 38 & $12.5(7.8-19.0)$ & & $14.4(2-28.7)$ & \\
\hline \multicolumn{6}{|l|}{ Smoking } \\
\hline Yes & 55 & $12.4(6.5-25.6)$ & \multirow[t]{2}{*}{0.583} & $14.7(3.8-42.3)$ & \multirow[t]{2}{*}{0.519} \\
\hline No & 25 & $12.5(4.4-19.0)$ & & $15.5(2-29.6)$ & \\
\hline \multicolumn{6}{|c|}{ Alcohol intake } \\
\hline Yes & 57 & $12.5(5.0-25.6)$ & \multirow[t]{2}{*}{0.675} & $14.4(4.2-42.3)$ & \multirow[t]{2}{*}{0.985} \\
\hline No & 23 & $12.4(4.4-17.5)$ & & $15.3(2-33.4)$ & \\
\hline \multicolumn{6}{|l|}{ TNM grade } \\
\hline $1+\|$ & 22 & $9.2(4.4-17.3)$ & \multirow[t]{2}{*}{0.0001} & $8.7(2.0-24.7)$ & \multirow[t]{2}{*}{0.002} \\
\hline$I I I+I V$ & 58 & $13.1(8.9-25.6)$ & & $15.7(2.8-42.60)$ & \\
\hline \multicolumn{6}{|c|}{ EBVNCA-lgA } \\
\hline$\leq 1: 160$ & 44 & $11.9(6.5-25.6)$ & \multirow[t]{2}{*}{0.693} & $14.9(4.2-33.4)$ & \multirow[t]{2}{*}{0.441} \\
\hline$\geq 1: 320$ & 36 & $12.6(4.4-19.0)$ & & $12.6(2.0-42.6)$ & \\
\hline
\end{tabular}

Notes: CTSB cathepsin B, CTSD cathepsin D, TNM tumor node metastasis, EBV Epstein-Barr virus, VCA-IgA viral capsid antigen IgA 


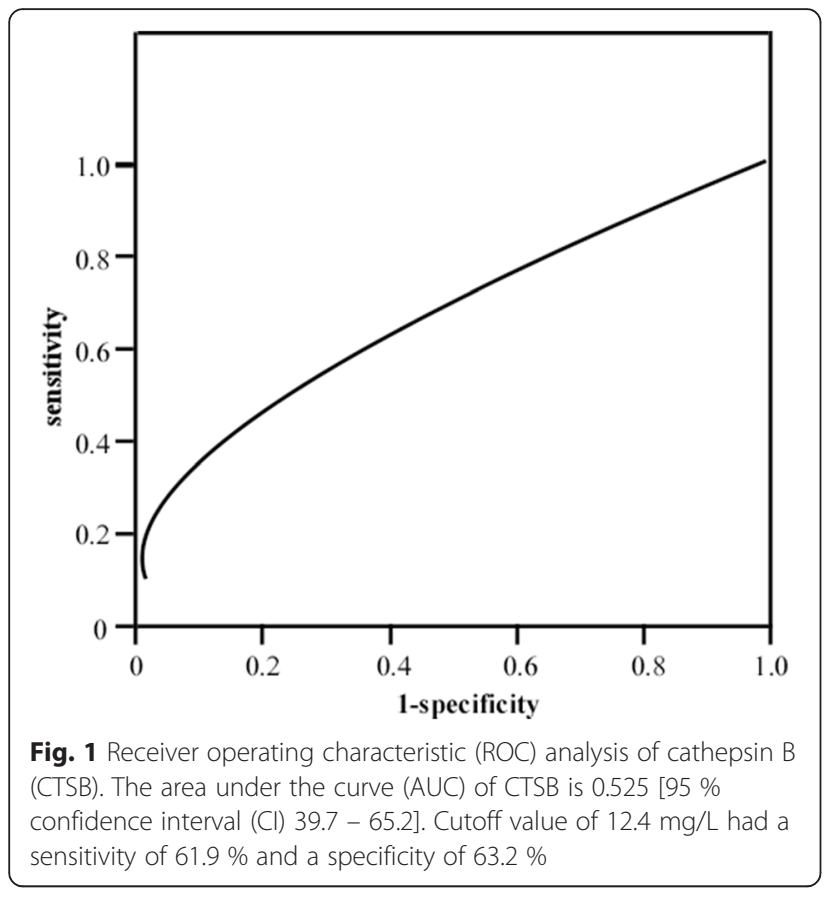

We hypothesize that CTSB and CTSD might serve as candidate biomarkers of NPC.

Retrospective immunohistochemical analysis showed that lung cancer patients with upregulated CTSB tend to have higher rates of hematogenous and intrapulmonary metastases [18]. CTSB activity is significantly elevated in a variant of B16 melanoma with tumor cells of high metastatic potential [19]. Intense expression of CTSD in high-grade carcinomas might be an indicator of invasive potential and aggressive behavior [20]. Among the patients with positive lymph nodes, those with tumor cells

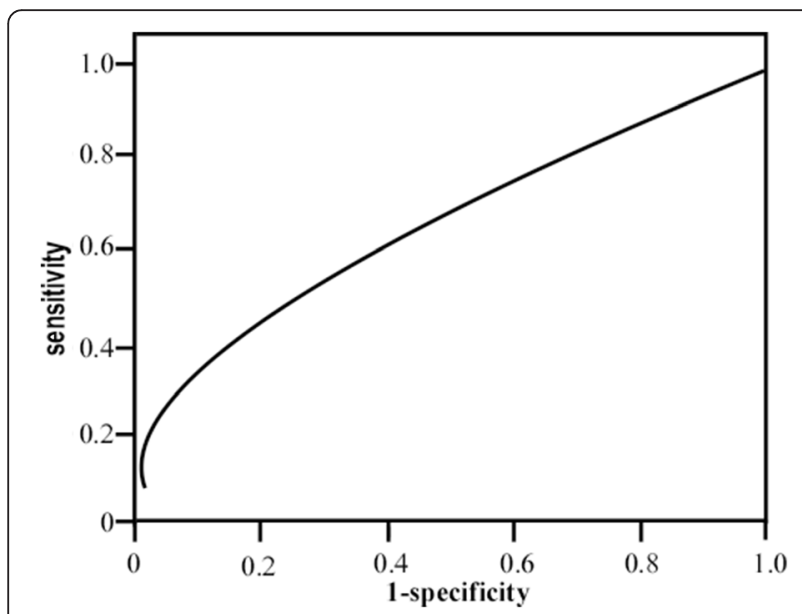

Fig. 2 Receiver operating characteristic (ROC) analysis of cathepsin D (CTSD). The area under the curve (AUC) of CTSD is 0.552 [95\% confidence interval (Cl) 42.3-68.1]. Cutoff value of $14.7 \mathrm{mg} / \mathrm{L}$ had a sensitivity of $66.7 \%$ and a specificity of $58.5 \%$
Table 4 Univariate and multivariate analyses of CTSB and CTSD for prognosis of patients with nasopharyngeal carcinoma

\begin{tabular}{|c|c|c|c|c|c|}
\hline \multirow[t]{2}{*}{ Variables } & \multirow[t]{2}{*}{ Number } & \multirow{2}{*}{$\begin{array}{l}\text { Median } \\
\text { PFS } \\
\text { (months) }\end{array}$} & \multirow{2}{*}{$\begin{array}{l}\text { Univariate analysis } \\
p \text { value }\end{array}$} & \multicolumn{2}{|c|}{$\begin{array}{l}\text { Multivariate } \\
\text { analysis }\end{array}$} \\
\hline & & & & $p$ value & RR \\
\hline \multicolumn{6}{|l|}{ CTSB } \\
\hline$>12.4 \mathrm{mg} / \mathrm{l}$ & 42 & 37.0 & 0.699 & 0.878 & 0.938 \\
\hline$\leq 12.4 \mathrm{mg} / \mathrm{l}$ & 38 & 37.8 & & & \\
\hline \multicolumn{6}{|l|}{ CTSD } \\
\hline$>14.7 \mathrm{mg} / \mathrm{l}$ & 39 & 35.9 & 0.216 & 0.341 & 1.524 \\
\hline$\leq 14.7 \mathrm{mg} / \mathrm{l}$ & 41 & 38.6 & & & \\
\hline \multicolumn{6}{|l|}{ Sex } \\
\hline Men & 54 & 37.2 & 0.902 & 0.566 & 1.286 \\
\hline Women & 26 & 35.8 & & & \\
\hline \multicolumn{6}{|l|}{ Age } \\
\hline$\leq 45$ years & 40 & 37.3 & 0.699 & 0.779 & 0.892 \\
\hline$>45$ years & 40 & 37.5 & & & \\
\hline \multicolumn{6}{|l|}{ TNM stage } \\
\hline $1+\|$ & 20 & 39.1 & 0.454 & 0.556 & 1.376 \\
\hline$I I I+I V$ & 60 & 36.4 & & & \\
\hline \multicolumn{6}{|l|}{ VCA-IgA } \\
\hline$\leq 1: 160$ & 45 & 37.5 & 0.735 & 0.769 & 1.265 \\
\hline$\geq 1: 320$ & 35 & 36.8 & & & \\
\hline \multicolumn{6}{|l|}{ Smoking } \\
\hline Yes & 55 & 36.6 & 0.913 & 0.991 & 0.995 \\
\hline No & 25 & 37.9 & & & \\
\hline
\end{tabular}

Notes: CTSB cathepsin B, CTSD cathepsin D, PFS progression-free survival, $R R$ relative risk, TNM tumor node metastasis, EBV Epstein-Barr virus, VCA-IgA viral capsid antigen-IgA

immunopositive for CTSD had a higher risk of relapse [21]. Determination of CTSD status in breast cancer patients might help identify those with different risk levels of relapse [22]. Our previous work suggests that CTSB and CTSD are associated with NPC metastasis [13]. Next, we analyzed whether CTSB and CTSD concentrations increase in the sera of metastatic NPC patients. The data showed that CTSB and CTSD levels were significantly higher in the sera of patients with metastatic NPC than in the sera of patients with no metastasis. Therefore, the detection of CTSB and CTSD concentrations might indicate the prognosis for NPC metastasis.

Epstein-Barr virus (EBV) infection is an important etiological factor for NPC [23]. EBV serological tests, including IgA for early antigen (EA) and VCA, have been used for NPC detection for a long time [24, 25]. We analyzed whether CTSB and CTSD concentrations are related to EBV infection in NPC patients. We did not observe any significant relationship between CTSB and CTSD concentrations, and EBV infection. We did not yet identify any relationship between CTSB and CTSD 
concentrations, and patient age, sex, alcohol intake, and smoking status.

CTSB and CTSD expression was detected in NPC and normal nasopharyngeal epithelial tissues, and the results showed that CSTB and CTSD expression levels were significantly higher in the NPC tissues compared to the normal nasopharyngeal epithelial tissues. Further analysis showed that the upregulation of CTSB or CTSD was significantly correlated with lymph node metastasis, advanced clinical stage, recurrence, distant metastasis, and poor prognosis $[13,26]$. Altogether, it is suggested that the serum concentrations of CTSB and CTSD might be potential biomarkers of NPC. Unfortunately, data enabling a definitive discrimination is currently not available.

\section{Conclusions}

In summary, serum CTSB and CTSD concentrations were significantly higher in patients with advanced NPC, indicating that they could be diagnostic biomarkers of NPC. CTSB and CTSD serum levels are of prognostic significance in NPC progression. Future prospective studies with larger patient numbers and longer followup periods are required to further evaluate the prognostic significance of these potential biomarkers.

\section{Abbreviations \\ AUC: area under the curve; Cl: confidence intervals; CTSB: cathepsin B; CTSD: cathepsin D; EA-IgA: IgA for early antigen; EBV: Epstein-Barr virus; ELISA: enzyme-linked immunosorbent assay; NPC: nasopharyngeal carcinoma; PBS: phosphate-buffered saline; PFS: progression-free survival; ROC: receiver operating characteristic; TNM: tumor node metastasis; VCA-IgA: viral capsid antigen IgA.}

\section{Competing interests}

The authors declare that they have no competing interest.

\section{Authors' contributions}

GJT and XWT performed ELISA assays and drafted the manuscript. QXL and TK selected the patients and collected the blood samples. YJL and JPL were responsible for statistical analyses and revised the manuscript. XMZ and FQT were involved in the study design and discussion of the results, as well as the critical review of the manuscript. All authors read and approved the final manuscript.

\section{Funding}

This work was supported by the National Natural Science Foundation of China (81372282, 81402368, 81402265, and 81502346), Natural Science Foundation of Guangdong (S2013010013360), Fundamental Research Funds for the Guangdong Province (2011B061300053), Zhuhai Best Medical Instrument Appliance Inc., and the Foundation of State Key Laboratory of Oncology in South China (HN2011-04).

\section{Author details}

'Department of Clinical Laboratory and Medical Research Center, Xiangya Hospital, Central South University, 87 Xiangya Road, Changsha 410008Hunan, China. ${ }^{2}$ Department of Clinical Laboratory and Medical Research Center, Zhuhai People's Hospital, Zhuhai Hospital of Jinan University, 79 Kangning Road, Zhuhai 519000Guangdong, China. ${ }^{3}$ Metallurgical Science and Engineering, Central South University, 21 Lushan South Road, Changsha 410083, China. ${ }^{4}$ State Key Laboratory of Oncology in South China, Sun Yat-sen University Cancer Center, Guangzhou, China.
Received: 26 April 2015 Accepted: 15 March 2016

Published online: 19 March 2016

\section{References}

1. Leung TW, Tung SY, Sze WK, Wong FC, Yuen KK, et al. Treatment results of 1070 patients with nasopharyngeal carcinoma: an analysis of survival and failure patterns. Head Neck. 2005;27:555-65.

2. Tatsumi-Tamori A, Yoshizaki T, Miwa T, Furukawa M. Clinical evaluation of staging system for nasopharyngeal carcinoma: comparison of fourth and fifth editions of UICC TNM classification. Ann Otol Rhinol Laryngol. 2000;109:1125-9.

3. Lee AW, Poon YF, Foo W, Law SC, Cheung FK, Chan DK, et al. Retrospective analysis of 5037 patients with nasopharyngeal carcinoma treated during 1976-1985: overall survival and patterns of failure. Int J Radiat Oncol Biol Phys. 1992;23:261-70.

4. Lv X, Xiang YQ, Cao SM, Qian CN, Li NW, Guo L, et al. Prospective validation of the prognostic value of elevated serum vascular endothelial growth factor in patients with nasopharyngeal carcinoma: more distant metastases and shorter overall survival after treatment. Head Neck. 2011;33:780-5.

5. Kuester D, Lippert $H$, Roessner A, Krueger $S$. The cathepsin family and their role in colorectal cancer. Pathol Res Pract. 2008;204:491-500.

6. Joyce JA, Hanahan D. Multiple roles for cysteine cathepsins in cancer. Cell Cycle. 2004;3:1516-9.

7. Kayser K, Richter N, Hufnagl P, Kayser G, Kos J, Werle B. Expression, proliferation activity and clinical significance of cathepsin B and cathepsin L in operated lung cancer. Anticancer Res. 2003;23:2767-72.

8. Tan GJ, Peng ZK, Lu JP, Tang FQ. Cathepsins mediate tumor metastasis. World J Biol Chem. 2013;4:91-101.

9. Mort JS, Buttle DJ. Cathepsin B. Int J Biochem Cell Biol. 1997;29:715-20.

10. Dean RT. Direct evidence of importance of lysosomes in degradation of intracellular proteins. Nature. 1975;257:414-16.

11. Fusek M, Vetvicka V. Dual role of cathepsin D: ligand and protease. Biomed Pap Med Fac Univ Palacky Olomouc Czech Repub. 2005;149:43-50.

12. Lively $\mathrm{S}$, Schlichter $\mathrm{LC}$. The microglial activation state regulates migration and roles of matrix-dissolving enzymes for invasion. J Neuroinflammation. 2013;10:1742-2094.

13. Li Y, Lu J, Peng Z, Tan G, Liu N, Huang D, et al. N,N'-dinitrosopiperazinemediated AGR2 is involved in metastasis of nasopharyngeal carcinoma. PLoS One. 2014;9(4):e92081.

14. Schmitt M, Janicke F, Moniwa N, Chucholowski N, Pache L, Graeff H. Tumorassociated urokinase-type plasminogen activator: biological and clinical significance. Biol Chem Hoppe Seyler. 1992;373:611-22.

15. Werle B, Kraft C, Lah TT, Kos J, Schanzenbacher U, Kayser K, et al. Cathepsin $B$ in infiltrated lymph nodes is of prognostic significance for patients with nonsmall cell lung carcinoma. Cancer. 2000;89:2282-91.

16. Guszcz T, Swieczkowska M, Milewska E, Gorodkiewicz E, Kozlowski R, Roszkowska-Jakimiec W. Cathepsin D and B activity in the serum of patients with urothelial bladder cancer. Pol Merkur Lekarski. 2014;36:386-8.

17. Kos J, Nielsen HJ, Krasovec M, Christensen IJ, Cimerman N, Stephens RW, et al. Prognostic values of cathepsin B and carcinoembryonic antigen in sera of patients with colorectal cancer. Clin Cancer Res. 1998:4:1511-16.

18. Fujise N, Nanashima A, Taniguchi Y, Matsuo S, Hatano K, Matsumoto Y, et al. Prognostic impact of cathepsin B and matrix metalloproteinase-9 in pulmonary adenocarcinomas by immunohistochemical study. Lung Cancer. 2000;27:19-26.

19. Sloane BF, Dunn JR, Honn KV. Lysosomal cathepsin B: correlation with metastatic potential. Science. 1981;212:1151-3.

20. Sivaranjini Y, Angadi P, Kumar G. Immuno-histochemical evaluation of Cathepsin D in malignant salivary gland carcinomas. Gulf J Oncolog. 2012;1:38.

21. Lentari I, Segas I, Kandiloros D. The importance of cathepsin's-D tissular detection in laryngeal squamous cell carcinoma. Acta Otorhinolaryngol Belg. 2002;56:383.

22. Markicevic M, Kanjer K, Mandusic V, Buta M, Neskovic-Konstantinovic Z, Nikolic-Vukosavljevic D. Cathepsin D as an indicator of clinical outcome in early breast carcinoma during the first 3 years of follow-up. Biomark Med. 2013;7:747-58.

23. Liu JP, Cassar L, Pinto A, Li H. Mechanisms of cell immortalization mediated by EB viral activation of telomerase in nasopharyngeal carcinoma. Cell Res. 2006;16:809-17.

24. Zeng $Y$, Zhang $L G$, Li HY, Jan $M G$, Zhang $Q$, Wu YC, et al. Serological mass survey for early detection of nasopharyngeal carcinoma in Wuzhou City. China Int J Cancer. 1982;29:139-41. 
25. Tsang RK, Vlantis AC, Ho RW, Tam JS, To KF, van Hasselt CA. Sensitivity and specificity of Epstein-Barr virus IGA titer in the diagnosis of nasopharyngeal carcinoma: a three-year institutional review. Head Neck. 2004;26:598-602.

26. Cheng AL, Huang WG, Chen ZC, Zhang PF, Li MY, Li F, et al. Identificating cathepsin $\mathrm{D}$ as a biomarker for differentiation and prognosis of nasopharyngeal carcinoma by laser capture microdissection and proteomic analysis. J Proteome Res. 2008;7:2415-26.

Submit your next manuscript to BioMed Central and we will help you at every step:

- We accept pre-submission inquiries

- Our selector tool helps you to find the most relevant journal

- We provide round the clock customer support

- Convenient online submission

- Thorough peer review

- Inclusion in PubMed and all major indexing services

- Maximum visibility for your research

Submit your manuscript at www.biomedcentral.com/submit
Biomed Central 